JOURNAL OF THE

AMERICAN MATHEMATICAL SOCIETY

Volume 21, Number 3, July 2008, Pages 847-862

S 0894-0347(08)00593-6

Article electronically published on February 12, 2008

\title{
SINGULARLY PERTURBED ELLIPTIC SYSTEMS AND MULTI-VALUED HARMONIC FUNCTIONS WITH FREE BOUNDARIES
}

\author{
L. A. CAFFARELLI AND FANG-HUA LIN
}

\section{INTRODUCTION}

The purpose of this paper is to study the solutions $u^{\varepsilon}=\left(u_{1}^{\varepsilon}, \ldots, u_{m}^{\varepsilon}\right)$ of a family of singularly perturbed systems of elliptic equations and their asymptotic limits.

The common characteristic of these problems is that in the singular limit, the components of the solutions of these systems group in different blocks and the supports of the different blocks become disjoint. In a special case [CLLL, CR, the limit gives rise to a multiple scalar-valued harmonic function, and a free boundary problem between components.

To illustrate the basic problems involved and our main results we start with two examples.

Example A. The simplest example, which appears in population dynamics and particle systems, concerns the case in which each component segregates from the other; see $[\mathrm{BS}, \mathrm{CR}$. In this case the $\varepsilon$-system reads as follows: In the domain $\Omega \subset R^{n}$, we consider the following system of equations:

$$
\begin{cases}\Delta u_{i}^{\varepsilon}=\frac{1}{\varepsilon} \sum_{j \neq i} u_{i} u_{j}, & \\ u_{i}^{\varepsilon}=f_{i} \geq 0 & \text { in } \partial \Omega, \\ f_{i} f_{j}=0 & \text { for } i \neq j .\end{cases}
$$

As $\varepsilon$ goes to zero, we expect the vector $u^{\varepsilon}$ to converge to a nonnegative solution vector (i.e., each component is nonnegative) $u=\left(u_{1}, \ldots, u_{m}\right)$ that satisfies $\left(\sup u_{i}\right)^{0} \cap\left(\sup u_{j}\right)^{0}=\varnothing$ and $\left.\Delta u_{i}\right|_{\left(\sup u_{i}\right)^{0}}=0($ see $[\mathrm{CR}])$. If we have only two components $u_{1}, u_{2}$, then $\Delta\left(u_{1}^{\varepsilon}-u_{2}^{\varepsilon}\right) \equiv 0$ and hence also for the limit $\Delta\left(u_{1}-u_{2}\right) \equiv 0$. That is, we can recover $u_{1}, u_{2}$ as $\omega^{+}, \omega^{-}$where $\omega$ is the harmonic function with data $f_{1}-f_{2}$ along $\partial \Omega$.

If the number of variables is larger (say three), then we may have $u_{1}$ extending into $-u_{2},-u_{2}$ into $u_{3}$, and $u_{3}$ into $-u_{1}$, and hence we need to repeat the cycle

Received by the editors August 24, 2006.

2000 Mathematics Subject Classification. Primary 35B25, 35P30, 49N60.

Key words and phrases. Singular limit, regularity of free interface, multiple-valued harmonic functions, harmonic maps.

(C)2008 American Mathematical Society Reverts to public domain 28 years from publication 
once more in order to again reach $u_{1}$. That is the case for the solution

$$
r^{3 / 2} \cos \frac{3}{2} \theta=\operatorname{Re}\left(z^{3 / 2}\right)
$$

that goes around the origin twice.

In general, in $\mathbb{R}^{n}$, what we should expect for the scalar-valued two-components limiting case is that one has an $n-2$ dimensional filament replacing the origin and a double-valued harmonic function that lives in the "two leaves" Riemann surface that goes around the "filament."

This is what we plan to prove in this paper, at least in the measure theoretical sense: There is a closed "singular set" $S$ of Hausdorff dimension $n-2$, where $|u|$ decays of order $|u(x)| \leq[d(x, S)]^{3 / 2}$; and outside of $S$, if $\left|u\left(x_{0}\right)\right|=0$, in a neighborhood of $x_{0}$ there are exactly two nonzero components, $\left(u_{1}, u_{2}\right)$, and $u_{1}-u_{2}$ is harmonic.

Example B. A more interesting and delicate problem occurs when the grouping is nontrivial. Such a problem arises in the theory of harmonic maps into singular spaces and also in a more general segregation of competing species; see CLLL, CTV3, CTV4.

In this case the limiting map $\left(u_{1}, \ldots, u_{m}\right)$ is supposed to be a map from $\Omega$ into a special set in $R^{m}$, which is not all of $R^{m}$ but is a disjoint family of orthonormal subspaces $R^{k_{1}}, R^{k_{2}}, \ldots, R^{k_{\ell}}$ of $R^{m}$ with

$$
\bigoplus R^{k_{j}}=R^{m}
$$

(e.g., a line and a plane). We expect that as before the different groups $\left(u_{1}, \ldots, u_{k_{1}}\right)$, $\left(u_{k_{1}+1}, \ldots, u_{k_{1}+k_{2}}\right)$, etc., will have disjoint supports.

The corresponding $\varepsilon$-system is the Euler-Lagrange equations for singularly perturbed variational integrals. For example, $u^{\varepsilon}$ 's are minimizers of

$$
\int_{\Omega}\left[\left|\nabla u^{\varepsilon}\right|^{2}+2 F_{\varepsilon}\left(u_{\varepsilon}\right)\right] d x
$$

where $F_{\varepsilon}(u)=\sum_{i<j} \frac{\beta_{i j}}{\varepsilon} u_{i}^{2} u_{j}^{2}$ with $\beta_{i j} \geq 0$ and $u^{\varepsilon}=f$ on the boundary of $\Omega$ such that each component of $f$ is nonnegative. We should point out that the nonnegativity of the components of $f$ immediately implies the nonnegativity of each of the components of $u^{\varepsilon}$. For most of the discussion in this article, we do not need this nonnegativity assumption, but it simplifies part of the arguments for the regularity of free boundaries between supports of components (or blocks of components) of a vector-valued solution $u^{\varepsilon}$. Otherwise, the zero set of each component of $u^{\varepsilon}$, which, as we shall see, at the limit becomes a nodal set of the corresponding components, also enters the picture, since at the limit each component of the solution is a harmonic function on the domain of its definition. Its structure and its geometric measure were studied much earlier; see [L3].

For the above problem, the picture we now have in mind is still pretty much the same as before: There is an $(n-2)$ dimensional closed filament $S$ where at least three supports of disjoint components converge, outside of which, near any other point $x_{0}$ for which $u\left(x_{0}\right)=0$, there are exactly two subdomains that are supports of two components (or two blocks) of the limiting solution $u$, say $\Omega_{k_{i}}, \Omega_{k_{j}}$, separated by a smooth free boundary. 
The main result of this paper states roughly that, away from this closed subset $S \subseteq \Omega$ of the Hausdorff dimension $\leq n-2$, the free interfaces between various components (or blocks of components) of $u$ are, in fact, $C^{1, \alpha}$ smooth hypersurfaces.

We point out that M. Conti et al. in [CTV, CTV2] studied similar singularly perturbed systems related to some optimal partition problem for nonlinear eigenvalues and Nehari type problems. Besides the case of two components, the behavior of solutions as well as the regularity of free interfaces were, however, not settled. One can easily apply our results to those problems studied in CTV, CTV2]. Recently in CTV3, CTV4 the Lipschitz regularity of the limiting solutions as well as the regularity of the free boundaries in the case of two dimensions were also established.

We also point out that, due to the special forms of the equations in Example A, we find that using the maximum principle and the viscosity solutions method is more effective for Example A, and that will be discussed in more detail in a forthcoming article [CL2]. The present article will be focused on Example B for which we use the variational method. For a special case of the limiting problem, each component becomes a scalar-valued function (no nontrivial grouping; the limits of these systems have already been studied, e.g., in (CLLL), and it becomes an optimal partition problem; see BBH, CTV, CTV2. For this latter problem, the authors have recently proved in $\mathrm{CL}$ the regularity of free interfaces of optimal partition problems for the eigenvalues of the Laplacian operator on $\Omega$ with the Dirichlet boundary conditions; see [CL].

Organization of the paper. This paper is organized as follows. We shall discuss only problems of the type from Example B. Problems of the type described in Example A will be studied in CL2. We start with some uniform estimates for a solution $u_{\varepsilon}$ of singularly perturbed systems, and they are discussed in Section 2 . These estimates allow one to pass to the limits to obtain the limiting free boundary problems. In Section 3 we introduce the Almgren monotonicity formula and discuss a few easy consequences. It gives some preliminary control on the vanishing order of the solutions as well as the Lipschitz continuity of solutions. We discuss homogeneous blow-ups and classifications of homogeneous degree 1 blow-ups in Section 4 . This leads to the flatness of free boundaries. The main regularity theorem for free boundaries is described in Section 4 and proved in Section 5.

\section{UNIFORM ESTIMATES FOR $u^{\varepsilon}$}

For problem (1.1), the uniform Hölder continuity of $u^{\varepsilon}$ (and in some cases uniform Lipschitz estimates) was established in CR]; see also CTV, CTV2 for related discussions. Here we present some uniform estimates for the minimizers of (1.2) that would be sufficient to allow us to pass to the limit.

Let $u_{\varepsilon}$ be a solution of $-\Delta u_{\varepsilon}+\operatorname{grad} F_{\varepsilon}\left(u_{\varepsilon}\right)=0$ in $\Omega \subseteq \mathbb{R}^{n}$. Here $u_{\varepsilon}=$ $\left(u_{\varepsilon}^{1}, \ldots, u_{\varepsilon}^{m}\right)$,

$$
\begin{gathered}
F_{\varepsilon}(u)=\frac{1}{\varepsilon} \sum_{i<j} \beta_{i j}\left(u^{i} u^{j}\right)^{2}, \quad u \in \mathbb{R}^{m}, \quad \varepsilon>0,0 \leq \beta_{i j} \leq \beta<\infty, \\
F_{\varepsilon}^{-1}\{0\}=\bigoplus_{j=1}^{\ell} R^{k_{j}}=\sum .
\end{gathered}
$$

We shall call the vector formed by components of $u$ with image in one of $R^{k_{j}}$ the $k_{j}{ }^{\text {th }}$ block of $u$. 
Lemma 2.1. Let

$$
E(r)=\frac{1}{r^{n-2}} \int_{B_{r}\left(x_{0}\right)}\left(\left|\nabla u_{\varepsilon}\right|^{2}+2 F_{\varepsilon}\left(u_{\varepsilon}\right)\right) d x .
$$

Then $E(r) \uparrow$ in $r$, for all $B_{r}\left(x_{0}\right) \subset \Omega$.

Proof. Let $x_{0}=0$. It suffices to verify that

$$
\left.\frac{d}{d r} E(r)\right|_{r=1} \geq 0
$$

whenever $B_{1}(0) \subseteq \Omega$. Note that by a scaling the general case $r>0$ can be reduced to this particular case, and it suffices to show that (2.1) with $F_{\varepsilon}$ may be replaced by $F_{\lambda}$, for some $\lambda>0$.

Let $u_{\varepsilon}^{r}(x)=u_{\varepsilon}(r x)$; then

$$
E(r)=\int_{B_{1}}\left[\left|\nabla u_{\varepsilon}^{r}(x)\right|^{2}+2 r^{2} F_{\varepsilon}\left(u_{\varepsilon}^{r}\right)\right] d x
$$

Thus

$$
\left.\frac{d}{d r} E(r)\right|_{r=1}=\int_{B_{1}}\left[2 \nabla u_{\varepsilon} \cdot \nabla \dot{u}_{\varepsilon}+4 F_{\varepsilon}\left(u_{\varepsilon}\right)+2 F_{\varepsilon}^{\prime}\left(u_{\varepsilon}\right) \cdot \dot{u}_{\varepsilon}\right] d x
$$

where $\dot{u}_{\varepsilon}=\left.\frac{d}{d r}\right|_{r=1} u_{\varepsilon}^{r}=x \cdot \nabla u_{\varepsilon}(x)$. Using the equation $-\Delta u_{\varepsilon}+F_{\varepsilon}^{\prime}\left(u_{\varepsilon}\right)=0$ and integrating by parts, one has

$$
\left.\frac{d}{d r} E(r)\right|_{r=1}=\int_{\partial B_{1}} 2 u_{\varepsilon r}^{2}+\int_{B_{1}} 4 F_{\varepsilon}\left(u_{\varepsilon}\right) \geq 0 .
$$

Let $e_{\varepsilon}(x)=\left|\nabla u_{\varepsilon}\right|^{2}+2 F_{\varepsilon}\left(u_{\varepsilon}\right)$.

We note that if we use Remark 2 below, then ( $\operatorname{set} u^{\varepsilon}=u$ below to simplify the notation)

$$
0 \leq \Delta \frac{u_{i}^{2}}{2}=\left|\nabla u_{i}\right|^{2}+\sum_{j \neq i} \beta_{i j} \frac{u_{i}^{2} u_{j}^{2}}{\varepsilon} .
$$

Since the energy bounds, we have $u_{\varepsilon}=u \in L_{\text {loc }}^{\infty}(\Omega)$. This combined with the above inequality implies, for any $B_{2 R}(x) \subset \Omega$,

$$
\int_{B_{R}(x)} \frac{e_{\varepsilon}(y)}{|x-y|^{n-2}} d y \leq C(R)\left\|u_{\varepsilon}\right\|_{L^{\infty}\left(B_{2 R}(x)\right)}^{2}<\infty .
$$

From (2.2), one deduces that

$$
\int_{0}^{R} \frac{E(r)}{r} d r \leq C(R)\left\|u_{\varepsilon}\right\|_{L^{\infty}\left(B_{2 R}\right)}^{2} .
$$

Here $E(r)$ is the normalizer energy defined in Lemma 2.1. Hence, for a suitable small $r_{0}$ one has $E\left(r_{0}\right) \leq \delta\left(n, C_{0}\right) \ll 1$. One can choose such $r_{0}$ uniformly on each compact subset of $\Omega$.

Suppose one has the following Bochner type inequality: $\Delta e_{\varepsilon}(x) \geq-c_{0} e_{\varepsilon}^{2}$ in $\Omega$. Then via an argument such as that in [Sc or [L, p. 77], one could derive the uniform Lipschitz estimate for $u_{\varepsilon}$. But at present, we are not able to verify the above Bochner type inequality. In other words, the uniform (in $\varepsilon$ ) Lipschitz continuity of solutions to singularly perturbed systems remains unknown, and the uniform Hölder estimates as presented in [CR still seem to be the best uniform regularity result for the solutions $u_{\varepsilon}$ in general. Similar issues also exist for the 
case $F_{\varepsilon}(u)=\frac{1}{\varepsilon} \sum_{i \neq j} u_{i} u_{j}\left(u_{i} \geq 0\right)$, but that will be discussed in CL2 where a different method is used as we mentioned in the Introduction.

Remark 1. As $\varepsilon$ goes to zero, since

$$
\Delta u_{i}^{+} \geq \frac{1}{\varepsilon} \sum_{j \neq i} u_{i}^{+} \beta_{i j} u_{j}^{2} \geq 0
$$

one deduces that

$$
\int_{B_{r}} \sum_{j \neq i} \frac{u_{i}^{+} \beta_{i j} u_{j}^{2}}{\varepsilon}=\int_{B_{r}} \Delta u_{i}^{+} \leq C r^{-2} \int_{B_{2 r}} u_{i}^{+} d x, \quad \text { for all } B_{2_{r}} \subset \Omega .
$$

Note the final quantity remains bounded independently of $\varepsilon$.

Remark 2. As in Remark 1, since

$$
0 \leq \frac{\Delta u_{i}^{2}}{2}=\left(\nabla u_{i}\right)^{2}+\sum_{j \neq i} \frac{u_{i}^{2} u_{j}^{2} \beta_{i j}}{\varepsilon}
$$

we have that

$$
\int_{B_{r}}\left[\left(\nabla u_{i}\right)^{2}+\sum_{j \neq i} \frac{u_{i}^{2} u_{j}^{2} \beta_{i j}}{\varepsilon}\right]
$$

is locally uniformly bounded by

$$
C^{-2} \int_{B_{2 r}}\left(u_{i}\right)^{2} d x, \quad \text { for all } B_{2_{r}} \subset \Omega .
$$

Lemma 2.2. Let $v, w$ be two nonnegative subharmonic functions in $B_{1}$. Assume that $0 \leq v, w \leq 1$ and

$$
\int_{B_{1}(0)} v w<\varepsilon
$$

Then $v(0)$ or $w(0)<\varepsilon^{\theta}$.

Proof. Suppose not, i.e., $v w(0)>C \varepsilon^{\theta}$. In particular both $v(0), w(0)>C \varepsilon^{\theta}$. On the other hand, we must have the Lebesgue measure of the sets to satisfy

$$
\left|\left\{v>\varepsilon^{1 / 4}\right\} \cap\left\{w>\varepsilon^{1 / 4}\right\}\right|<\varepsilon^{1 / 2} .
$$

In particular, for any ring $\Gamma_{k}=B_{2^{-k}} \backslash B_{2^{-(k+1)}}$ for which $2^{-n k}>C \varepsilon^{1 / 2}$, we have that either

$$
\left|\left\{v>\varepsilon^{1 / 4}\right\}\right| \text { or } \quad\left|\left\{w>\varepsilon^{1 / 4}\right\}\right|<\frac{3}{4}\left|\Gamma_{k}\right| \text {. }
$$

Therefore, in each of these rings, if we start from the bound $v, w \leq 1$ on $B_{1}$, the maximum of $\left(v-\varepsilon^{1 / 4}\right)^{+}$or $\left(w-\varepsilon^{1 / 4}\right)^{+}$decreases by a fixed constant (from the mean value theorem) multiple from $B_{2^{-k}}$ to $B_{2^{-(k+1)}}$. But there are $|\log \varepsilon|$ of these rings, therefore either $v(0)$ or $w(0)<\varepsilon^{\theta}$ for some small $\theta$.

If we apply the above lemma to the square of components of the solutions $u^{\varepsilon}$, we obtain the following.

Corollary 2.3. $u_{i}^{\varepsilon} u_{j}^{\varepsilon}$ converge uniformly to zero, i.e.,

$$
\left(u_{i}^{+\varepsilon} u_{j}^{+\varepsilon}\right) \leq \varepsilon^{\theta}
$$

whenever $\beta_{i j}$ is positive. 
Corollary 2.4. $\int_{B_{r}} F_{\varepsilon}\left(u_{\varepsilon}\right) d x$ converges to zero.

Proof. Since either $\left|u_{i}\right|$ or $\left|u_{j}\right| \leq \varepsilon^{\theta}$,

$$
\frac{1}{\varepsilon} \sum_{i<j} u_{i}^{2} u_{j}^{2} \beta_{i j} \leq C \varepsilon^{\theta} \sum_{i<j} \frac{\left(u_{i}^{ \pm} u_{j}^{2}+u_{j}^{ \pm} u_{i}^{2}\right) \beta_{i j}}{\varepsilon} .
$$

Therefore, by Remark 1, one has

$$
\int_{B_{r}} F_{\varepsilon}\left(u_{\varepsilon}\right) \leq C \varepsilon^{\theta} .
$$

Corollary 2.5. The limiting vector $u$ satisfies

(a) $u_{i} u_{j} \equiv 0$ if $i \neq j$ and $\beta_{i j}$ is positive.

(b) $u_{i}$ is harmonic in the interior of the set $D_{i}=\left\{u_{j}=0\right.$ for $\left.\beta_{i j}>0\right\}$.

\section{Almgren's monotonicity formula and its CONSEquences}

We start by discussing a classical monotonicity formula for multiple-valued harmonic functions due to Almgren [A]. In the simplest case, that of a harmonic function, it reads: Let $u$ be harmonic in $B_{1}, u(0)=0$; then

$$
N(r)=\frac{r D(r)}{H(r)} \nearrow \quad \text { in } r
$$

where

$$
D(r)=\int_{B_{r}}|\nabla u|^{2} \quad \text { and } \quad H(r)=\int_{\partial B_{r}} u^{2} .
$$

Note that a similar fact remains true for solutions to a much wider class of second order elliptic equations; see GL.

In our case, we must substitute $u^{2}=|u-0|^{2}$ by the distance in the singular space $\sum \equiv F_{\varepsilon}^{-1}\{0\} \equiv \bigoplus_{j=1}^{\ell} R^{k_{j}}$ where $u$ lives; i.e., $d_{\Sigma}(p, q)=|p-q|^{2}$ if both $p$ and $q$ are in the same $R^{k_{j}}$, and $d_{\Sigma}(p, q)=|p|+|q|$ if $p$ and $q$ are in different $R^{k_{j}}$ 's.

Suppose $u: \Omega \subseteq \mathbb{R}^{n} \rightarrow \Sigma$ is a finite energy minimizing harmonic map and let $p=u\left(x_{0}\right)$; then

$$
D(r)=\int_{B_{r}\left(x_{0}\right)}|\nabla u|^{2} d x, \quad H(r)=\int_{\partial B_{r}\left(x_{0}\right)} d_{\Sigma}^{2}(u(x), p) .
$$

\section{Lemma 3.1.}

for $r \in\left(0, d\left(x_{0}, \partial \Omega\right)\right)$.

$$
N(r)=\frac{r D(r)}{H(r)} \nearrow \quad \text { in } r
$$

Proof. We follow the same proof as for the harmonic functions case; see GL].

The usual energy monotonicity formula for harmonic maps (say energy minimizing) says that

$$
\frac{d}{d r}\left(\int_{B_{r}}|\nabla u|^{2} d x / r^{n-2}\right)=\frac{2 \int_{\partial B_{r}} u_{\rho}^{2}}{r^{n-1}} \geq 0
$$

(see $[\mathrm{Sc}])$. Next, by a direct calculation, one has

$$
\Delta_{x} d_{\Sigma}^{2}(u(x), p) \geq 2|\nabla u|^{2}(x) .
$$


Integrating by parts, one derives from (3.2) that

$$
\frac{d}{d r} H(r)=\frac{n-1}{r} H(r)+\int_{\partial B_{r}} \frac{d}{d \rho} d_{\Sigma}^{2}(u(x), p) \geq \frac{n-1}{r} H(r)+2 D(r) .
$$

(3.1) and (3.3) imply that $N^{\prime}(r) \geq 0$.

An important feature of Almgren's monotonicity formula is that it controls the "degree" at the origin of the "first" homogeneous term in the Taylor expansions. For instance, if $u$ is a homogeneous harmonic polynomial $P$ of degree $k$, then

$$
N(r) \equiv k .
$$

So the monotonicity of $N(r)$ implies on one hand that oscillation "increases with" $r$, but on the other, since, in the case of a harmonic function $N(0)=N\left(0, x_{0}\right)$ corresponds to the degree of the leading Taylor polynomial of $u$ at $x_{0}$, this implies semicontinuity: If for instance

$$
N\left(r, x_{0}\right) \leq k+\frac{1}{2}
$$

since $N(r, x)$ is continuous in $x$, we have $N(r, x) \leq k+\frac{3}{4}$ for $x$ close to $x_{0}$, and thus $N(0, x) \leq k+\frac{3}{4}$ for $x$ near $x_{0}$ (see [GL). That is, the order of a zero $x_{0}$ of a harmonic function is upper-semicontinuous in $x_{0}$.

The following corollary was proved in some cases in [CTV3] and [CTV4] for such limiting problems; also see CL2 for a different proof.

Corollary 3.2. $u$ is Lipschitz.

Proof. We have that $u$ is approximate differentiable almost everywhere in the $L^{2}$ sense [L2, p. 36]. At any such point $x$, where $u$ is approximate differentiable, $N(0, x) \geq 1$; hence by the upper-semicontinuity of the vanishing order, $N\left(0^{+}, x_{0}\right) \geq$ 1 for any $x_{0}$. But

$$
\frac{d}{d r} \log \left(\frac{H(r)}{r^{n-1}}\right) \geq \frac{2 N(r)}{r} \geq \frac{2 N\left(0^{+}\right)}{r},
$$

therefore

$$
\frac{H(r)}{r^{n-1}} \leq \frac{H(R)}{R^{n-1}}\left(\frac{r}{R}\right)^{2}
$$

However, for $R=1$,

$$
H(1)=\int_{\partial B_{1}} d^{2}(u(x), p) \leq C .
$$

Since $d^{2}$ is subharmonic, this implies

$$
\left.d^{2}\right|_{B_{r / 2}(p)} \leq \frac{H(r)}{r^{n-1}} \leq C r^{2} .
$$

Lemma 3.3. The set where all $u_{i}$ 's vanish has empty interior unless $u \equiv 0$.

Proof. It is an easy consequence of a much stronger result that could be drawn from the Almgren monotonicity formula; see GL. 


\section{Flatness of the phase transition}

In the previous sections we verified the convergence of energy minimizers of the variational integrals

$$
\int_{\Omega}\left(\left|\nabla u_{\varepsilon}\right|^{2}+2 F_{\varepsilon}\left(u_{\varepsilon}\right)\right) d x
$$

with given Dirichlet boundary condition $\left.u_{\varepsilon}\right|_{\partial \Omega}=f$ as $\varepsilon \rightarrow 0$. Here $f$ is a map from $\partial \Omega$ into $\Sigma$. The limits are energy minimizing harmonic maps $u: \Omega \rightarrow \Sigma$ with the same Dirichlet boundary condition $\left.u\right|_{\partial \Omega}=f$ such that $u$ is locally uniformly Lipschitz continuous inside $\Omega$. Our aim in this section and the next is to study the set $\Gamma=\{x \in \Omega: u(x)=\underline{0}\}$. As we noted in Lemma 3.3. one can deduce that the Lebesgue measure of $\Gamma$ is zero unless $u \equiv 0$.

To avoid some additional complications from the nodal sets of harmonic functions given by various components of $u$, we should simply consider the case when each component of $u$ is nonnegative. Note that this is the case for various applications; see CR, CLLL, CTV, CTV2]. Such nonnegativity of components of $u$ can be easily deduced when the Dirichlet boundary value $f$ also verifies such conditions.

We first want to establish a gap in the values of the vanishing order function $N(x)$ for $x \in \Gamma$; that is, as pointed out before, the smallest possible value of $N(x)$ is 1 , which we shall show corresponds to the linear behavior of each component of $u$ near $x$, and the next one is $N(x)=1+\delta_{n}$ for some positive dimensional constant $\delta_{n}$. Here for convenience, we let $N\left(u, x_{0}, r\right)$ be as defined in Lemma 3.1, and $N\left(u, x_{0}\right)=\lim _{r \rightarrow 0^{+}} N(u, x, r)$. In the case where the dependence of $u$ is clear, we should simply drop $u$ in the above notation; instead we have have $N\left(x_{0}\right)$ and $N\left(x_{0}, r\right)$.

Lemma 4.1. For each $x_{0} \in \Gamma$, let

$$
N\left(x_{0}\right)=\lim _{r \rightarrow 0} N\left(x_{0}, r\right) .
$$

Then either $N\left(x_{0}\right)=1$ or $N\left(x_{0}\right) \geq 1+\delta_{n}$ for a positive dimensional constant $\delta_{n}$.

Proof. Let $x_{0} \in \Gamma$; thus $u\left(x_{0}\right)=\underline{0} \in \mathbb{R}^{m}$. Define

$$
u_{\lambda}(x)=\frac{u\left(x_{0}+\lambda x\right)}{\left\|u\left(x_{0}+\lambda x\right)\right\|_{L^{2}\left(B_{1}\right)}}, \quad x \in B_{1}(0) \subseteq \mathbb{R}^{n}, \quad \lambda \text { is a positive small number. }
$$

It is easy to check that the corresponding function $N\left(u_{\lambda}, x_{0}, r\right)$ for $u_{\lambda}$ satisfies $N\left(u_{\lambda}, 0, r\right)=N\left(u, x_{0}, \lambda r\right)$. Thus as $\lambda \rightarrow 0^{+}$, one has $u_{\lambda}(x)=\bar{u}(x)$ weakly in $B_{2}(0)$ up to a subsequence; see [L3]. For $\bar{u}$, which is an energy minimizing harmonic map from $B_{1}$ into $\Sigma$, one has $N(\bar{u}, 0, r) \equiv N\left(x_{0}\right) \geq 1$. We remark that the minimum value of $N(x)$ is 1 , because that is the case in a dense set (in fact, at all differentiable points of $u$, which is a.e. by Rademacher's theorem), and $N(x)$ is upper-semicontinuous.

It is now easy to check (from the proof of Lemma 3.1) that $\bar{u}$ is homogeneous of degree $N\left(x_{0}\right)$; that is, $\bar{u}(x)=r^{\alpha} g(\omega)$, where $\omega=\frac{x}{|x|} \in \mathbb{S}^{n-1}$ and $\alpha=N\left(x_{0}\right)$. Hence we want to show that either $\alpha=1$ or $\alpha \geq 1+\delta_{n}$ for some $\delta_{n}>0$. We consider two possibilities:

(i) If the image of $g\left(\mathbb{S}^{n-1}\right)$ lies in at least three distinct components (those $\mathbb{R}^{k_{j}}$ 's) in $\Sigma$, then $\mathbb{S}^{n-1}$ would divide into at least three subdomains (the pre-images of these distinct components of $\Sigma$ ). There is at least one such subdomain $A$ that has the volume $A \leq \frac{1}{3}$ volume of $\mathbb{S}^{n-1}$. Since $\bar{u}$ is a homegoneous harmonic map from 
$B_{1}^{n}(0)$ into $\Sigma, g(\omega)$ must be an eigenfunction (vector) of the Laplacian $\Delta_{\mathbb{S}^{n-1}}$ on $A$ with zero Dirichlet boundary condition; that is,

$$
\Delta_{\mathbb{S}^{n-1}} g+\lambda g=0 \in A \text { and } g=0 \text { on } \partial A .
$$

By the separation of variables, one has $\alpha^{2}+(n-2) \alpha-\lambda=0$. A simple eigenvalue estimate (using symmetrization) yields $\lambda \geq \lambda_{n} \geq n-1+\eta_{n}$ for some positive dimensional constant $\eta_{n}$. Thus $\alpha \geq 1+\delta_{n}$ follows.

(ii) If the image $g(\omega)$ is contained in the union of at most two components of $\Sigma$, then if $g(\omega)$ is always contained in one component of $\Sigma, \bar{u}$ is simply a harmonic function (may be vector valued). Thus the vanishing order $N\left(x_{0}\right)$ is either 1 or not less than 2.

If the image of $g(\omega)$ lies in exactly two components of $\Sigma$, then $\mathbb{S}^{n-1}$ would divide into exactly two components $A_{1}$ and $A_{2}$ so that $g(\omega)$ would be an eigenvector of $\Delta_{\mathbb{S}^{n-1}}$ on $A_{1}$ and $A_{2}$, respectively, with zero Dirichlet boundary conditions; that is,

$$
\left\{\begin{array}{ll}
\Delta_{\mathbb{S}^{n-1}} g+\mu_{j} g=0 & \text { in } A_{j}, \\
g=0 & \text { on } \partial A_{j},
\end{array} \text { for } j=1,2 .\right.
$$

One must have $\mu_{1}=\mu_{2}$ since both give the characteristic exponent $\alpha$ (the homogeneity of $\bar{u}$ ).

We note that we only consider the case when each component of $u$ is nonnegative (and hence would be true for each component of $\bar{u}$ and $g$ ). Suppose $g_{j}, 1 \leq j \leq k$, are components of $g$ supported in $A_{1}$, and $g_{j}, k+1 \leq j \leq \ell$, are those components of $g$ supported in $A_{2}$. Then, using the nonnegativity (and the simplicity of the first eigenfunctions of $\Delta_{\mathbb{S}^{n-1}}$ on subdomains of $\mathbb{S}^{n-1}$ ), we may conclude that $g_{j}=\lambda_{j} g_{1}$ for $1 \leq j \leq k$, and $g_{j}=\lambda_{j} g_{k+1}$ for $k+1 \leq j \leq \ell$. For convenience, we assume that $g_{1}$ and $g_{k+1}$ are not identically zero.

Sub-Lemma 4.1. The function $v_{1}=\left(\sum_{j=1}^{k} \lambda_{j}^{2}\right)^{1 / 2} \bar{u}_{1}$ extends crossing $\partial \Omega_{1} \cap \partial \Omega_{2}$ harmonically into $-v_{2}=-\left(\sum_{k+1}^{\ell} \lambda_{j}^{2}\right)^{1 / 2} \bar{u}_{k+1}$. Here $\Omega_{1}$ and $\Omega_{2}$ are cones over $A_{1}$ and $A_{2}$ with vortex at the origin $\underline{0}$.

The proof of the lemma follows simply from the fact that $v_{1}$ and $v_{2}$ are harmonic, respectively, in $\Omega_{1}$ and $\Omega_{2}$. Moreover, since $\bar{u}=r^{\alpha} g(w)$ is an energy minimizing harmonic map from $\bar{B}_{1}=\bar{\Omega}_{1} \cup \bar{\Omega}_{2}$ into $\Sigma$, and the image of $g\left(\mathbb{S}^{n-1}\right)$ is contained in two half-line segments, the first variation calculation leads to $\left|\frac{\partial v_{1}}{\partial \nu}\right|^{2}=\left|\frac{\partial v_{2}}{\partial \nu}\right|^{2}$ on $\partial \Omega_{1} \cap \partial \Omega_{2}$ in the pointwise sense. Thus if one defines

$$
v= \begin{cases}v_{1} & \text { in } \Omega_{1}, \\ -v_{2} & \text { in } \Omega_{2},\end{cases}
$$

$v$ is harmonic in $B_{1}$ in a weak sense.

From Sub-Lemma 4.1 one concludes that $\alpha$ must be 1 , and thus $\Omega_{1}$ and $\Omega_{2}$ are half-balls in $B_{1}^{n}$.

Remark. If $\mathbb{S}^{n-1}$ is divided into two subdomains $A_{1}$ and $A_{2}$ such that $\Delta_{\mathbb{S}^{n-1}}$ has the same first eigenvalues on both $A_{1}$ and $A_{2}$ with respect to the Dirichlet boundary conditions, that is, $\mu_{1}=\mu_{2}$, and if the corresponding characteristic values are the same $\alpha=1$, that is, $\mu_{1}=\mu_{2}=$ first eigenvalue on the hemisphere of $\Delta_{\mathbb{S}^{n-1}}$ with respect to the zero Dirichlet boundary conditions as in our case, then $A_{1}$ and $A_{2}$ are hemispheres. This follows by a symmetrization argument. 
Corollary 4.2. If $N\left(x_{0}\right)>1$ for $x_{0} \in \Gamma$, then $N\left(x_{0}\right) \geq \gamma_{0}$, the eigenvalue of the spherical cap of volume $\leq \frac{1}{3}$ volume of $\mathbb{S}^{n-1}$; in particular, $\gamma_{0} \geq \frac{3}{2}$.

Corollary 4.3. If $N\left(x_{0}\right)=1$, components of $\bar{u}$ are simply given by

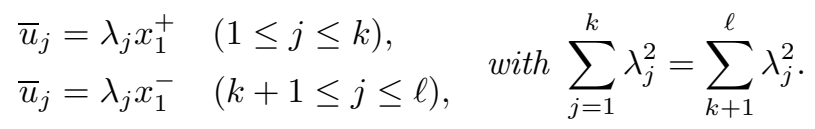

We define $\Gamma^{*}=\{x \in \Gamma: N(x)=1\}$. We start with the following observation. If $x_{o} \in \Gamma^{*}$, then $N\left(x_{0}\right)=1$, and then there is a $\delta_{0}>0$ such that for all $x \in B_{\delta_{0}}\left(x_{0}\right) \cap \Gamma$, one has $N(x) \leq 1+\delta_{n / 2}$. This follows from the upper-semicontinuity of $N(x)$ in $\Omega$. By Lemma 4.1, one must have $N(x) \equiv 1$ for all $x \in \Gamma \cap B_{\delta_{0}}\left(x_{0}\right)$; that is, $\Gamma \cap B_{\delta_{0}}\left(x_{0}\right) \subseteq \Gamma^{*}$ or equivalently $\Gamma^{*}$ is relatively open in $\Gamma$.

Next we want to investigate the property of $\Gamma^{*}$ near a point $x_{0} \in \Gamma^{*}$. Since $N\left(x_{0}\right)=1$, each homogeneous blow-up of $u$ at $x_{0}$ (that is a subsequential limit of $u_{\lambda}(x)$ as $\lambda \rightarrow 0^{+}$) is of degree 1 . However, such homogeneous blow-ups may not be unique.

For each $\vec{e} \in \mathbb{S}^{n-1}$, we let $T(e, \varepsilon)$ be the $\varepsilon$-strip in the $\vec{e}$-direction passing the origin $\underline{0}$, that is,

$$
T(\vec{e}, \varepsilon)=\left\{x \in B_{1}^{n}(0):|x \cdot \vec{e}| \leq \varepsilon\right\} .
$$

Lemma 4.4 (Flatness of the free boundary). Let $u: B_{2} \rightarrow \sum$ be an energy minimizing map with $f_{\partial B_{2}}|u|^{2}=1$. Then for every $\varepsilon \in(0,1)$, there is a $\delta(\varepsilon)>0$ such that if $N(\underline{0}, 2) \leq 1+\delta(\varepsilon), \underline{0} \in \Gamma^{*}$, then $u$ can be well-approximated by linear functions in $B_{1}^{n} \mid T(e, \varepsilon)$ for some $\vec{e} \in \mathbb{S}^{n-1}$ (cf. Corollary 4.3). Here $u$ is wellapproximated by linear functions in $B_{1}^{n}(0) \mid T(e, \varepsilon)$ means

$$
\begin{gathered}
\left|u(x)-\ell_{\varepsilon}^{ \pm}(x)\right|_{C^{1}} \leq \varepsilon \quad \text { for } x \in B_{1}^{ \pm}(0) \backslash T(e, \varepsilon), \\
B_{1}^{ \pm}(0)=\left\{x \in B_{1}(0): x \cdot \vec{e} \lessgtr 0\right\}, \quad \text { and for some } \\
\ell_{\varepsilon}^{ \pm}(x)=\vec{a}_{\varepsilon}^{ \pm}(x \cdot \vec{e}) \quad \text { where } \vec{a}_{\varepsilon}^{+}=\left(\lambda_{1}, \ldots, \lambda_{k}\right), \vec{a}_{\varepsilon}^{-}=\left(\lambda_{k+1}, \ldots, \lambda_{\ell}\right) .
\end{gathered}
$$

Proof. The conclusion of the above lemma follows easily by a contradiction argument. One notes that the homogeneous degree 1 map $u: B_{2}^{n}(0) \rightarrow \Sigma$ must be given by a two-component linear map (see the proof of Corollary 4.3).

We note that due to normalization one can assume $1 \leq\left|\vec{a}_{\varepsilon}^{ \pm}\right| \leq 3$.

Remark. (a) Let $u: B_{2}^{n}(0) \rightarrow \Sigma$ be an energy minimizing harmonic map such that $N(\underline{0}, 2) \leq 1+\delta, \underline{0} \in \Gamma^{*}$. Then for all $x \in \Gamma \cap B_{2 / 3}^{n}(\underline{0})$, one has $N(x, 1) \leq 1+c(\delta)$. Here $c(\delta) \rightarrow 0^{+}$as $\delta \rightarrow 0+$.

(b) From (a) one concludes: If $u: B_{2} \rightarrow \Sigma$ is energy minimizing with $N(\underline{0}, 2) \leq$ $1+\delta, \underline{0} \in \Gamma^{*}$, then for any $x \in \Gamma \cap B_{2 / 3}^{n}(0), 0<r<1$, one has $N(x, r) \leq$ $1+c(\delta)$. In particular, when $\delta$ is sufficiently small, a proper scaled of $u$ at every point $x \in \Gamma \cap B_{2 / 3}^{n}(\underline{0})$, and for every scale $\lambda, 0<\lambda<1$, can be well-approximated by two linear functions in $B_{\lambda}(x) \backslash\left\{x+T\left(e_{\lambda}, \varepsilon \lambda\right)\right\}$; cf. Corollary 4.3 .

More precisely we have the following.

Corollary 4.5. For any $\varepsilon>0$, there is a $\delta(\varepsilon)>0$, such that if $u: B_{2} \rightarrow \Sigma$ is harmonic and $N(\underline{0}, 2) \leq 1+\delta(\varepsilon)$, then for any $0<r<1$, the function $u_{x, r}(y)=$ 
$\frac{u(x+r y)}{\|u(x+r y)\|_{L^{2}\left(B_{1}\right)}}, x \in B_{2 / 3}$, can be well-approximated by two linear vector-valued functions

$$
\ell_{r}^{ \pm}(y)=\vec{a}_{r}^{ \pm} y \cdot \vec{e}_{r}
$$

in $y \in B_{1}(0) \backslash T\left(e_{r}, \varepsilon\right)$, for some $\vec{e}_{r} \in \mathbb{S}^{n-1}$, and $\vec{a}_{r}^{ \pm}$are as given in Lemma 4.4 with $\frac{1}{3} \leq\left|a_{r}^{ \pm}\right| \leq 3$. Here $\left\|u_{x, r}(y)\right\|_{L^{2}\left(B_{1}\right)}=1$.

Theorem 4.6. Under the same assumptions as those in Lemma 4.4, there is $\theta_{0}=$ $\theta_{0}(n) \in(0,1)$ such that $\Gamma \cap B_{\theta_{0}}(\underline{0})$ is a bi-Hölder embedded topological $(n-1)$ dimensional ball.

Proof. It is clear from the conclusion of Lemma 4.4 and the remark above that, for all $x \in \Gamma \cap B_{2 / 3}(0), 0<r<1, \Gamma \cap B_{r}(x)$ is contained in a strip $T\left(\vec{e}_{r}, \varepsilon r\right)$ passing the point $x$, for some $\varepsilon=\varepsilon(\delta) \ll 1$, so long as one assumes $\delta$ is sufficiently small. Moreover, for each pair of points $Y_{1}, Y_{2}$ in $B_{r}^{+}(x) \backslash T\left(\vec{e}_{r}, \varepsilon r\right)$ and $B_{r}^{-}(x) \backslash T\left(\vec{e}_{r}, \varepsilon r\right)$, respectively, so that $\pi_{e}\left(Y_{1}\right)=\pi_{e}\left(Y_{2}\right)$, where $\pi_{e}: \mathbb{R}^{n} \rightarrow\left\{x \in \mathbb{R}^{n}: x \cdot \vec{e}=0\right\}$ is the orthogonal projection, there is a point on the $\overline{Y_{1} Y_{2}}$ segment which is in $B_{r}(x) \cap \Gamma$ (cf. Lemma 4.1). Therefore, the Hausdorff distance between $\Gamma \cap B_{r}(x)$ and $T\left(\vec{e}_{r}, \varepsilon r\right) \cap B_{r}(x)$ is less than $2 \varepsilon r$. In other words, $\Gamma \cap B_{2 / 3}(0)$ satisfies the Reifenberg condition (see [M]). Reifenberg's topological disc theorem implies that $\Gamma \cap B_{\theta_{0}}(0)$, for some $\theta_{0}(n)>0$, is a bi-Hölder image of $B_{\theta_{0}}^{n-1}(0)$.

In the next section we shall show a "clean-up lemma" that says, mainly, that if $N(\underline{0}, 1) \leq 1+\delta$ for a small enough $\delta$, then on $B_{1 / 2}$ there are exactly two components, say $\Omega_{1}$ and $\Omega_{2}$, separated by a $C^{1, \alpha}$ surface $S=\partial \Omega_{1} \cap \partial \Omega_{2}$.

We are now ready to show the interface regularity. Our main theorem is the following:

Theorem 4.7. (a) If $N\left(0^{+}\right)=1$ at $x_{0}$, then in a neighborhood of $x_{0}, B_{r}\left(x_{0}\right)$, there are exactly two components $\Omega_{1}, \Omega_{2}$, and $\partial \Omega_{1} \cap \partial \Omega_{2}$ is a $C^{1, \alpha}$ hypersurface.

(b) The closed set where $N\left(0^{+}\right)>1$ has Hausdorff dimension $n-2$.

Proof of Part (b). Let $\Gamma=\{x \in \Omega: u(x)=0\}, \Gamma^{*}=\{x \in \Gamma: N(x)=1\}$. Then we have shown that for any $x \in \Gamma \mid \Gamma^{*}, N(x) \geq 1+\delta_{n}$ for some $\delta_{n}>0$. Since $N(x)$ is upper-semicontinuous in $x$, it is then easy to see that $\Gamma \mid \Gamma^{*}$ is closed in $\Omega$. We want to show that the Hausdorff dimension of $\Gamma \mid \Gamma^{*} \leq n-2$.

For this purpose, we shall adopt the notions $N(x, u)=N(x), \Gamma_{u}, \Gamma_{u}^{*}$, etc., to represent their dependence on $u$. We first note that, for a ball $B_{R}\left(x_{0}\right) \subset \Omega$, the set of all harmonic maps ( $\neq$ constant) $u$ from $B_{R}\left(x_{0}\right)$ into the singular space $\Sigma$ with $N\left(u, x_{0}, R\right) \leq N_{0}$, for some $N_{0} \in R_{+}$, is compact in the following sense. If $\left\{u_{i}\right\}$ is a sequence of such harmonic maps from $B_{R}\left(x_{i}\right)$ into $\Sigma$ with $N\left(u_{i}, x_{0}, R\right) \leq N_{0}<\infty$, then there is a subsequence $\left\{u_{i}^{\prime}\right\}$ such that $u_{i}^{\prime} \hookrightarrow u_{\infty}$ weak ${ }^{*}$ in $W_{\text {loc }}^{1, \infty}\left(B_{R}\left(x_{0}\right)\right) \cap$ $H_{\text {loc }}^{1}\left(B_{R}\right)$, such that $u_{\infty}: B_{R}\left(x_{0} \rightarrow \Sigma\right.$ is a harmonic map with $N\left(u_{\infty}, x_{0}, R\right) \leq N_{0}$. In particular $u_{\infty} \neq$ constant.

Next, we consider the family of relative closed subsets of $B_{R}\left(x_{0}\right), S_{u} \equiv \Gamma_{u} \backslash$ $\Gamma_{u}^{*} \equiv\left\{x \in B_{R}\left(x_{0}\right): N(x, u) \geq 1+\delta_{n}\right\}$, for $u: B_{R}\left(x_{i}\right) \rightarrow \Sigma$ harmonic and $N\left(u, x_{0}, R\right) \leq N_{0}$. From the compactness of such harmonic maps, we see that this family $\mathcal{F}=\left\{E\right.$ closed, $\left.E \subset S_{u}\right\}$ is compact in the Hausdorff metric (see, e.g., L2, pp. 56-57]). 
We are now in the position to apply the Federer dimension reduction principle to show that $S_{u}$ has Hausdorff dimension $\leq n-2$, for each $S_{u} \in \mathcal{F}$.

We assume, without loss of generality, that $R=1$ and $x_{0}=0$ (via a suitable normalization). Then we easily observe that $\mathcal{F}$ has the following two properties:

(i) If $E \in \mathcal{F}, E_{x, \lambda}=\frac{E-x}{\lambda} \cap B_{1}(0) \in \mathcal{F}$ for all $x \in B_{1}$ and $0<\lambda<1$.

(ii) Let $E_{i} \in \mathcal{F}$ and $E_{i} \rightarrow E_{*}$ in the Hausdorff distance; then $E_{*} \in \mathcal{F}$ (see, e.g., [L2, pp. 56-58]).

Finally we check that for $n=1, \mathcal{F}=\{\varnothing\}$. Indeed, if there is a solution of $u_{x x}=0$ on an interval with $u(a)=u_{x}(a)=0$, for some $a$ in this interval, then $u \equiv 0$. Therefore, by applying the dimension reduction principle (see [L2, pp. 49$52]$ ), we conclude that for $n=2$, each $S_{u} \in \mathcal{F}$ consists of isolated points, and for general $n \geq 3$, the Hausdorff dimension of $S_{u} \leq n-2$.

Remark. One can also show directly that for $n=2$, each $S_{u} \in \mathcal{F}$ consists of isolated points as follows. Consider the measurable function $g(\zeta)=\left(u_{x}^{2}-u_{y}^{2}\right)-2 i u_{x} \cdot u_{y}$; then it is well-known (see $\left[\mathrm{Sc}\right.$ ) that $g$ is holomorphic in $B_{1}$. Thus either $g \equiv 0$ or the zeros of $g$ are isolated. The case $g \equiv 0$ would imply that $u$ is conformal. The conclusion that $S_{u}$ is isolated follows. In fact, using the compactness of $\mathcal{F}$, one can locally bound the number of points of $S_{u}$ in $\Omega$.

\section{Proof of Theorem 4.7(a)}

The proof is divided into two key steps.

Step 1. If $N\left(0^{+}\right)$is close to 1 (hence it has to be 1), then there is an $r_{0}>0$ such that $B_{r_{0}}(0)=\bar{\Omega}_{1} \cup \bar{\Omega}_{2}$ with $u\left(\bar{\Omega}_{j}\right)$ are contained in some $\mathbb{R}^{k_{j}^{\prime}}$ component of $\sum=\bigoplus_{j=1}^{\ell} R^{k_{j}}$ for $j=1,2$.

This step is accomplished by the so-called "clean-up" lemma below. From this step and discussions in the previous section (in particular Corollary 4.5 and Theorem 4.6), one can easily verify that both $\Omega_{1}$ and $\Omega_{2}$ are the so-called NTA domains; see [JK].

Step 2. To show that the quotient $\frac{\partial u_{i}}{\partial \nu} / \frac{\partial u_{j}}{\partial \nu}$ is Hölder continuous for any two nonvanishing components of the limiting map $u$ supported in a given $\Omega_{\ell}(\ell=1,2)$. Moreover, we can renormalize the jump conditions of $\left|\frac{\partial v_{1}}{\partial \nu}\right|$ and $\left|\frac{\partial v_{2}}{\partial \nu}\right|$ in such a way that they are almost equal to 1 in a geometrically decaying sequence of balls $B_{2^{-k}}\left(x_{0}\right)$ for all $x_{0}$ in $B_{r_{0} / 2}(\underline{0})$, rendering the free boundary a $C^{1, \alpha}$ graph.

In some sense Step 2 would be a consequence of the boundary Harnack inequality (for NTA domains) and some other facts for which we shall offer a more direct argument.

Lemma 5.1 (Clean-up). Let $\underline{0} \in \Gamma^{*}$ and let $u$ be as in Lemma 4.4. Then u maps $B_{1}(0)$ into at most two different components of $\Sigma$. In other words, one may assume that $u_{j}(x) \equiv 0$ for all $u_{j}$ in other components of $\Sigma$.

Proof. Since $\underline{0} \in \Gamma^{*}$ and $N(0,2) \leq 1+\delta$, Lemma 4.4 implies that there are exactly two distinct components $L_{1}, L_{2}$ in $\Sigma$ such that $u\left(B_{3 / 2}(\underline{0}) \backslash T(\vec{e}, \varepsilon)\right) \subseteq L_{1} \cup L_{2}$, for an $\varepsilon=\varepsilon(\delta) \rightarrow 0^{+}$as $\delta \rightarrow 0^{+}$. Moreover, for every $x \in \Gamma \cap B_{1}(\underline{0})$, one has $u\left(B_{1 / 2}(x) \backslash T(\vec{e}, \varepsilon)\right) \subseteq L_{1} \cup L_{2}$. 
Thus, to verify the conclusion of Lemma [5.1, it suffices to show the following statement: If $\underline{0} \in \Gamma^{*}, N(0,1 / 2) \leq 1+\tilde{\delta}$ for a sufficiently small $\tilde{\delta}$, and

$$
u\left(B_{1 / 2}(\underline{0}) \backslash T(\vec{e}, \varepsilon)\right) \subseteq L_{1} \cup L_{2}
$$

for some $\varepsilon=\varepsilon(\tilde{\delta})$ and $\vec{e} \in \mathbb{S}^{n-1}$, where $L_{1}$ and $L_{2}$ are two distinct components in $\Sigma$, then for any $r \in(0,1 / 2)$,

$$
u\left(B_{r}(0) \backslash T(\vec{e}, \varepsilon r)\right) \subseteq L_{1} \cup L_{2}
$$

for the same two components $L_{1}$ and $L_{2}$ in $\Sigma$.

The above fact follows easily from the following observation. For each $r \in(0,1)$, there will be two distinct lines $L_{1}^{r}$ and $L_{2}^{r}$ in $\Sigma$, such that $u\left(B_{r}(0) \backslash T(\vec{e}, \varepsilon r)\right) \subseteq$ $L_{1}^{r} \cup L_{2}^{r}$. The problem is, of course, that such $L_{1}^{r}$ and $L_{2}^{r}$ may be dependent on $r$ in general. However, it is obvious that $L_{1}^{r}$ and $L_{2}^{r}$ depend on $r$ continuously. There are finitely many choices of such $L_{1}^{r}$ and $L_{2}^{r}$ in $\Sigma$, thus the choice of $L_{1}^{r} \cup L_{2}^{r}$ will be fixed for all $0<r<1$ by the continuity. The conclusion of the clean-up lemma follows.

Remark. As a consequence of the above proof, one also has the "uniqueness of images of homogeneous blow-up maps of degree 1". That is, for each $x \in \Gamma^{*}$, let $u_{*}$ be a homogeneous blow-up of $u$ at $x$, and suppose $u_{*}\left(B_{1}(0)\right) \subseteq L_{1} \cup L_{2}$ for two distinct components $L_{1}, L_{2}$ in $\Sigma$. Then for any homogeneous blow-up $\widetilde{u}$ of $u$ at $x$, $\widetilde{u}\left(B_{1}\right) \subset L_{1} \cup L_{2}$.

Remark. There is another way to look at the clean-up lemma, Lemma 5.1. In fact, from Lemma 4.4 and Corollary [4.5, it is not hard to see that there are two components $\Sigma_{1}, \Sigma_{2}$ in $\Sigma$, and two subdomains $\Omega_{1}=\Omega_{+}, \Omega_{2}=\Omega_{-}$of $B_{1}(\underline{0})$ such that for any $x_{0} \in B_{1 / 2}(\underline{0})$ with $u\left(x_{0}\right)=\underline{0}$ and $0<r<\frac{1}{2}$,

$$
D_{r}^{ \pm} \equiv B_{r}\left(x_{0}\right) \cap\left\{\left\langle x-x_{0}, \nu\right\rangle^{ \pm} \geq \varepsilon r\right\} \subset \Omega_{ \pm}
$$

for some unit vector $\nu=\nu\left(x_{0}, r\right)$, where $u\left(\Omega_{1}\right) \subseteq \Sigma_{1}, u\left(\Omega_{2}\right) \subseteq \Sigma_{2}$. We want to show that there is no such $\Omega_{3} \subseteq B_{1}(\underline{0})$, that $u\left(\Omega_{3}\right) \subseteq \Sigma_{3}$ (another component of $\Sigma)$, and that $\Omega_{3} \cap B_{1 / 2} \neq \varnothing$. Otherwise we would be able to find $0 \leq v \leq 1$ subharmonic in $B_{1}$ so that $v \equiv 0$ on the set $\left\{\left|\left\langle x-x_{0}, \nu\left(x_{0}, \frac{1}{2}\right)\right\rangle\right| \geq \varepsilon\right\} \cap B_{1 / 2}\left(x_{0}\right)$ for any $x_{0} \in B_{1 / 2}$ with $v\left(x_{0}\right)=\underline{0}$; thus in $B_{1 / 4}\left(x_{0}\right), 0 \leq v \leq e^{-c / \varepsilon}$. In particular, for a very large $M, v \leq 2^{-M}$ on $B_{1 / 4}\left(x_{0}\right)$. As a consequence, for any $x_{0} \in B_{1 / 2}$ with $v\left(x_{0}\right)=\underline{0}$ one has

$$
\sup _{B_{2}-k} v \leq 2^{-M k}
$$

via a simple iteration, and the properties of $\Omega_{1}, \Omega_{2}$ described above. Therefore

$$
\int_{B_{2-k-1}\left(x_{0}\right)} \Delta v d x \leq c_{0} 2^{(-M+2) k}
$$

and since we may assume $(M-2)>n+1$, we have that $\Delta v \equiv 0$ in $B_{1 / 2}(\underline{0})$. Thus $v \equiv 0$ in $B_{1 / 2}(\underline{0})$ by the maximum principle.

From the "clean-up" lemma and Theorem 4.6, one see that $B_{1}^{n}(0)=\Omega_{1} \cup \Omega_{2}$, $\Omega_{1}$ and $\Omega_{2}$ are separated by $\Gamma$ which satisfies the Reifenberg flatness condition. It is then relatively easy to check that $\Omega_{1}$ and $\Omega_{2}$ are NTA domains in the sense that they satisfy both corkscrew and Harnack chain conditions; see [JK]. 
Proof of Step 2. We adapt some of the notation introduced in the proof of SubLemma 4.1. In particular, we denote the support of $u_{j}$ by $\bar{\Omega}_{1}$ for $1 \leq j \leq k$ and the support of $u_{j}$ by $\bar{\Omega}_{2}$ for $k+1 \leq j \leq \ell$. Here $B_{1}(0)=\bar{\Omega}_{1} \cup \bar{\Omega}_{2}$. By [JK], one concludes that $\frac{u_{j_{1}}}{u_{j_{2}}}$ is Hölder continuous in $\bar{\Omega}_{1}$ (respectively, in $\bar{\Omega}_{2}$ ) whenever $1 \leq j_{1}, j_{2} \leq k\left(k+1 \leq j_{1}, j_{2} \leq \ell\right.$, respectively $)$.

From Corollary 4.3, we can define, for $x_{0} \in \partial \Omega_{1}\left(=\partial \Omega_{2}\right) \cap B_{1}(0)$, the quantities $\lambda_{j}\left(x_{0}\right), j=1, \ldots, \ell$, such that

$$
\sum_{j=1}^{k} \lambda_{j}^{2}\left(x_{0}\right)=\sum_{j=k+1}^{\ell} \lambda_{j}^{2}\left(x_{0}\right) .
$$

Similarly, we can define $u^{+}=v_{1}, u^{-}=v_{2}$ as in Sub-Lemma 4.1. After a normalization we can assume $\left|\frac{\partial u^{+}}{\partial \nu}\right|^{2}=\left|\frac{\partial u^{-}}{\partial \nu}\right|^{2}=1$ at a given point $x_{0}$ (say the origin) on $\Gamma=\partial \Omega_{j}(j=1,2)$. Moreover,

$$
|| \frac{\partial u^{+}}{\partial \nu}|-| \frac{\partial u^{-}}{\partial \nu}||(x) \leq c\left|x-x_{0}\right|^{\alpha}
$$

for $x \in \Gamma \cap B_{1 / 2}\left(x_{0}\right)$, by [JK] and Corollary 4.3.

Our final goal is to prove that in the balls, for some $0<r_{0}<1, B_{r_{0}^{k}}\left(x_{0}\right)$, $k=2,3 \ldots, u^{+}-u^{-}$becomes geometrically close to its harmonic replacements

$$
\begin{cases}\Delta h_{k}=0 & \text { in } B_{r_{0}^{k}}\left(x_{0}\right) \\ h_{k}=u^{+}-u^{-} & \text {on } \partial B_{r_{0}^{k}}\left(x_{0}\right) .\end{cases}
$$

This latter statement further implies that the measurements of the flatness of $\partial \Omega^{ \pm}$ decrease geometrically, and hence the $C^{1, \beta}$ regularity of $\Gamma=\partial \Omega^{ \pm}$.

To begin, we consider a harmonic function in $B_{1}$ so that $\left|h-t x_{1}\right|_{L^{\infty}\left(B_{1}\right)} \leq \varepsilon$ for some $\frac{1}{2} \leq t \leq 1$. Then, in $B_{1 / 2}$,

- $\left|\nabla h-t \vec{e}_{1}\right|(x) \leq c \varepsilon \quad \forall x \in B_{1 / 2}$.

- $\left|D^{2} h\right|(x) \leq c \varepsilon \quad \forall x \in B_{1 / 2}$.

- The level set $\{h=0\} \cap B_{1 / 2}$ is analytic.

- In $B_{r_{0}}$ (for a suitably small $r_{0}>0$ ) for some linear function $\ell,|h-\ell|(x) \leq$ $c \varepsilon r_{0}^{2}$.

- After a renomalization to a ball of radius $1,|\widetilde{h}-\widetilde{\ell}|_{L^{\infty}\left(B_{1}\right)} \leq c \varepsilon r_{0} \leq \frac{\varepsilon}{4}$.

In the last step the renormalization from $B_{r}$ to $B_{1}$ of a function $f$ is simply the function $\widetilde{f}(x)=\frac{1}{r} f(r x)$.

Keeping these simple observations in mind, we proceed with our proof of Step 2.

We note that in $B_{k}=B_{r_{0}^{k}}\left(x_{0}\right)$ the function $\sigma^{+}=\left(1+c r_{0}^{k \alpha}\right) u_{+}-u_{-}$is subharmonic. This follows from the facts that both $0 \leq u_{ \pm}$are harmonic in $\Omega_{ \pm}$ and $\left|\frac{\partial u_{-}}{\partial \nu}\right| \leq\left|\frac{\partial u_{+}}{\partial \nu}\right|+c r_{0}^{k \alpha}$ with $u_{ \pm}=0$ on $\Gamma=\partial \Omega_{ \pm}$. Similarly the function $\sigma^{-}=\left(1+c r_{0}^{k \alpha}\right) u_{-}-u_{+}$is also subharmonic in $B_{k}$. Therefore the harmonic extension $h_{k}$ of $u_{+}-u_{-}$on $B_{k}$ satisfies

$$
\left|h_{k}-\left(u_{+}-u_{-}\right)\right|_{L^{\infty}\left(B_{k}\right)} \leq c r_{0}^{k \alpha}\left\|u_{ \pm}\right\|_{L^{\infty}\left(B_{k}\right)} .
$$

In particular, $\left|h_{k+1}-h_{k}\right|_{L^{\infty}\left(B_{k+1}\right)} \leq c r_{0}^{k \alpha}\left\|u_{ \pm}\right\|_{L^{\infty}\left(B_{k}\right)}$. The Lipschitz regularity of $u$ implies that $\left\|u_{ \pm}\right\|_{L^{\infty}\left(B_{k}\right)} \leq c r_{0}^{k}$. Thus $\left\|\widetilde{h}_{k+1}-\widetilde{h}_{k}\right\|_{L^{\infty}\left(B_{\left.r_{0}\right)}\right.} \leq c_{*} r_{0}^{k \alpha}$ after the renormalization, which makes the ball $B_{k}$ become the unit ball. 
It is now clear that if $\widetilde{h}_{k}$ differs from a linear function $\widetilde{\ell}_{k}$ by $\varepsilon_{k}$ on $B_{1}$, then $\widetilde{h}_{k+1}$ differs from a linear function $\widetilde{\ell}_{k+1}$ on $B_{1}$ (where we consider all functions being renormalized to be defined on $B_{1 / r_{0}}$ ) by the amount $c \varepsilon_{k} r_{0}+c r_{0}^{k \alpha-1}$; that is,

$$
\left|\widetilde{h}_{k+1}-\widetilde{\ell}_{k+1}\right|_{L^{\infty}\left(B_{1}\right)} \leq c \varepsilon_{k} r_{0}+c r_{0}^{k \alpha-1} \text {. }
$$

Since one may initially assume that $|h-\ell|_{L^{\infty}\left(B_{1}\right)} \leq \varepsilon$ for any given positive $\varepsilon>0$, one concludes from the iterative estimates above that, for any given $\varepsilon_{0}>0$, one may find $r_{0}$ and $\varepsilon$ sufficiently small so that one has for $h_{0}=h, \ell_{0}=\ell, h_{k}, \ell_{k}, k \geq 1$, as above,

$$
\left|\widetilde{h}_{k}-\tilde{\ell}_{k}\right|_{B_{1}} \leq \varepsilon_{0} 2^{-k}, \quad k=1,2, \ldots
$$

Here $\widetilde{h}_{k}, \widetilde{\ell}_{k}$ are renormalizations from $B_{r_{0}^{k}}$ to $B_{1 / r_{0}}$.

We therefore conclude that

$$
\left|v-\ell_{k}\right|_{L^{\infty}\left(B_{r_{0}}^{k+1}\right)} \leq r_{0}^{k}\left[\varepsilon_{0} 2^{-k}+c r_{0}^{k \alpha}\right] \quad \text { where } \quad v(x)= \begin{cases}u_{+}(x), & x \in \Omega_{+}, \\ -u_{-}(x), & x \in \Omega_{-} .\end{cases}
$$

Thus the measurements of the flatness of $\Gamma=\{x: v(x)=0\}$ decrease geometrically on balls $B_{r_{0}^{k}}\left(x_{0}\right), k=1,2, \ldots$, for every $x_{0} \in B_{1 / 2}(\underline{0}) \cap \Gamma$; hence $\Gamma$ is of class $C^{1, \beta}$ for some $\beta>0$, and our proof is complete.

\section{ACKNOWLEDGEMENT}

The research of both authors was partially supported by NSF grants.

\section{REFERENCES}

[A] Almgren, F. J., Jr. $Q$ valued functions minimizing Dirichlet's integral and the regularity of area minimizing rectifiable currents up to codimension two. Bull. Amer. Math. Soc. (N.S.) 8 (1983), no. 2, 327-328. MR684900 (84b:49052)

[BBH] Bucur, D.; Buttazzo, G.; Henrot, A. Existence results for some optimal partition problems. Adv. Math. Sci. Appl. 8 (1998), no. 2, 571-579. MR.1657219 (99k:49004)

[BS] Burke, S. P.; Schuman, T. E. W. Diffusion flames. Industr. Eng. Chem. 20 (1928), no. 10, 998-1004.

[BD] Buttazzo, G.; Dal Maso, G. Shape optimization for Dirichlet problems: relaxed formulation and optimality conditions. Appl. Math. Optim. 23 (1991), no. 1, 17-49. MR.1076053 (92e:49055)

[Bda] Buttazzo, G.; Dal Maso, G. An existence result for a class of shape optimization problems. Arch. Rational Mech. Anal. 122 (1993), no. 2, 183-195. MR1217590(94i:49052)

[BZ] Bucur, D.; Zolésio, J.-P. $N$-dimensional shape optimization under capacitary constraint. J. Differential Equations 123 (1995), no. 2, 504-522. MR1362884 (97g:49054)

[CL] Caffarelli, L. A.; Lin, F. An optimal partition problem for eigenvalues. J. Sci. Comput. 31 (2007), no. 1, 5-18. Available online at: http://www.springerlink.com/content/ v671661832u5/?p=d2c1296d08c347ee80e9718c28995186 \&pi=4. MR 2304268

[CL2] Caffarelli, L. A.; Lin, F. Viscosity approach to a partition problem, preprint

[CLLL] Chang, S.-M.; Lin, C.-S.; Lin, T.-C.; Lin, W.-W. Segregated nodal domains of twodimensional multispecies Bose-Einstein condensates. Phys. D 196 (2004), nos. 3-4, 341361. MR.2090357 (2005g:82074)

[CR] Caffarelli, L. A.; Roquejoffre, J.-M. Uniform Hölder estimates in a class of elliptic systems and applications to singular limits in models for diffusion flames. Arch. Ration. Mech. Anal. 183 (2007), no. 3, 457-487. MR2278412

[CTV] Conti, M.; Terracini, S.; Verzini, G. Nehari's problem and competing species systems. Ann. Inst. H. Poincaré Anal. Non Linéaire 19 (2002), no. 6, 871-888. MR1939088 (2003i:35084)

[CTV2] Conti, M.; Terracini, S.; Verzini, G. An optimal partition problem related to nonlinear eigenvalues. J. Funct. Anal. 198 (2003), no. 1, 160-196. MR1962357(2004h:35171) 
[CTV3] Conti, M.; Terracini, S.; Verzini, G. Asymptotic estimates for the spatial segregation of competitive systems. Adv. Math. 195 (2005), no. 2, 524-560. MR2146353(2006b:35077)

[CTV4] Conti, M.; Terracini, S.; Verzini, G. A variational problem for the spatial segregation of reaction-diffusion systems. Indiana Univ. Math. J. 54 (2005), no. 3, 779-815. MR 2151234 (2006b:35180)

[GL] Garofalo, N.; Lin, F.-H. Monotonicity properties of variational integrals, $A_{p}$ weights and unique continuation. Indiana Univ. Math. J. 35 (1986), no. 2, 245-268. MR833393 (88b:35059)

[JK] Jerison, D. S.; Kenig, C. E. Boundary behavior of harmonic functions in nontangentially accessible domains. Adv. in Math. 46 (1982), no. 1, 80-147. MR676988 (84d:31005b)

[L] Lin, F.-H. Static and moving vortices in Ginzburg-Landau theories. Nonlinear partial differential equations in geometry and physics (Knoxville, TN, 1995), 71-111. Progr. Nonlinear Differential Equations Appl., 29. Birkhäuser, Basel, 1997. MR.1437152 (97m:35241)

[L2] Lin, F.; Yang, X. Geometric measure theory - an introduction. Advanced Mathematics (Beijing/Boston), 1. Science Press, Beijing; International Press, Boston, 2002. MR2030862 (2005a:28001)

[L3] Lin, F.-H. Nodal sets of solutions of elliptic and parabolic equations. Comm. Pure Appl. Math. 44 (1991), no. 3, 287-308. MR1090434 (92b:58224)

[M] Morrey, C. B., Jr. Multiple integrals in the calculus of variations. Die Grundlehren der mathematischen Wissenschaften, 130. Springer, New York, 1966. MR0202511 (34:2380)

[Sc] Schoen, R. M. Analytic aspects of the harmonic map problem. Seminar on nonlinear partial differential equations (Berkeley, Calif., 1983), 321-358. Math. Sci. Res. Inst. Publ., 2. Springer, New York, 1984. MR765241 (86b:58032)

[Sv] Šverák, V. On optimal shape design. J. Math. Pures Appl. (9) 72 (1993), no. 6, 537-551. MR.1249408 (94j:49047)

[Z] Ziemer, W. P. Weakly differentiable functions. Sobolev spaces and functions of bounded variation. Graduate Texts in Mathematics, 120. Springer, New York, 1989. MR1014685 (91e:46046)

Department of Mathematics, University of Texas at Austin, Austin, Texas 78712

E-mail address: caffarel@math.utexas.edu

Department of Mathematics, Courant Institute of Mathematical Sciences, New York University, 251 Mercer Street, New York, New York 10012

E-mail address: $\operatorname{linf} @$ cims.nyu.edu 